\title{
Polyominoes on Twisted Cylinders
}

\author{
Gill Barequet \\ Center for Geometric Computing \\ Dept. of Computer Science \\ The Technion-Israel Institute of Technology \\ Haifa 32000, Israel \\ barequet@cs.technion.ac.il
}

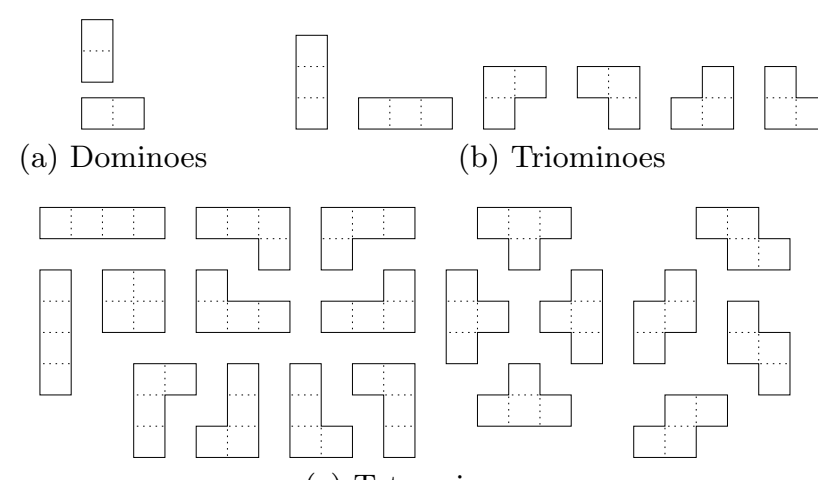

(c) Tetrominoes

Figure 1: All fixed dominoes, triominoes, and tetrominoes in the plane.

\begin{abstract}
In this video we show how to enumerate polyominoes on twisted cylinders, and explain how to use them for setting lower bounds on the asymptotic growth rate of polyominoes in the plane.
\end{abstract}

\section{Categories and Subject Descriptors}

G.2.1 [Mathematics of Computing]: Discrete Mathematics-Combinatorics

\section{Keywords}

Polyominoes; polycubes

\section{INTRODUCTION}

A polyomino of size $n$ is an edge-connected set of $n$ cells on the square lattice $\mathbb{Z}^{2}$. In statistical physics, polyominoes and their higher-dimensional generalizations (polycubes) play an important role in computing the mean cluster density in percolation processes, such as fluid flow in random media [3], and in modeling the collapse of branched polymer molecules in dilute solution [10]. Two fixed polyominoes are considered distinct if they differ in shape or orientation. Figure 1 shows all the fixed polyominoes of sizes two, three, and four. The number of fixed polyominoes of size $n$ is usually denoted

Copyright is held by the author/owner(s).

SoCG'13, June 17-20, 2013, Rio de Janeiro, Brazil.

ACM 978-1-4503-2031-3/13/06.

\author{
Mira Shalah \\ Center for Geometric Computing \\ Dept. of Computer Science \\ The Technion-Israel Institute of Technology \\ Haifa 32000, Israel \\ mshalah@cs.technion.ac.il
}

by $A(n)$. There are two long-standing open problems related to the study of polyominoes.

1. The enumeration of polyominoes, that is, finding a formula for $A(n)$ or computing $A(n)$ for specific values of $n$; and

2. Computing the quantity $\lim _{n \rightarrow \infty} \sqrt[n]{A(n)}$, the asymptotic growth rate of polyominoes (also known as "Klarner's constant").

To date, no formula is known for $A(n)$. Redelmeier [8] introduced the first efficient algorithm for counting polyominoes, in the sense that it generated all polyominoes sequentially without repetitions. The best known method (in terms of running time) for counting fixed polyominoes is a transfer-matrix algorithm suggested by Jensen in 2001. By running a parallel version of his algorithm, Jensen [5] computed $A(n)$ up to $n=56$.

Klarner [6] showed in a seminal work in 1967 that the limit $\lambda:=\lim _{n \rightarrow \infty} \sqrt[n]{A(n)}$ exists. Only three decades later did Madras [9] show that $\lim _{n \rightarrow \infty} A(n+1) / A(n)$ also exists and, hence, is equal to $\lambda$. At the present time not even a single significant decimal digit of $\lambda$ is known. The best-known lower [2] and upper [7] bounds on $\lambda$ are 3.9801 and 4.6496, respectively. It is generally assumed (see, e.g., [4]), as a conclusion from numerical methods applied to the known values of $A(n)$, that $\lambda \approx 4.06 \pm 0.02$. Jensen [5] refined this analysis, estimating $\lambda$ at $4.0625696 \pm 0.0000005$.

\section{TWISTED CYLINDERS}

Twisted cylinders were introduced in the context of polyominoes in [2]. For a fixed natural number $w$, the twisted cylinder of width (or perimeter) $w$ is the surface obtained from the Euclidean plane by identifying all pairs of points of the form $(x, y),(x-k w, y+k)$, for $k \in \mathbb{Z}$. Figure 2 shows a twisted cylinder of width 3 and a polyomino of size 9 embedded on it.

In [2], twisted cylinders were used in order to set a lower bound on $\lambda$. It was shown that for any $w \geq 1$, the constant $\lambda_{w}$, the asymptotic growth rate of polyominoes on a twisted cylinder of width $w$, is a lower bound on $\lambda$. It was also shown that the sequence $\left(\lambda_{w}\right)$ is monotone increasing. Elements of the sequence were computed up to $\lambda_{22}=3.9801 \ldots$, setting this bound on $\lambda .^{1}$ It was also proven [1] that the sequence $\left(\lambda_{w}\right)$ converges to $\lambda$.

\footnotetext{
${ }^{1}$ Recently, we computed $\lambda_{23}=3.9856 \ldots$, thereby improved the lower bound on $\lambda$.
} 


\begin{tabular}{|c|c|c|} 
& & \\
\hline 13 & 14 & 15 \\
\hline 10 & 11 & 12 \\
\hline 7 & 8 & 9 \\
\hline 4 & 5 & 6 \\
\hline 1 & 2 & 3 \\
\hline
\end{tabular}

(a) Cylinder

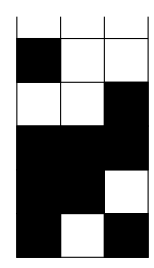

$\{1,3,4,5,7,8,9,12,13\}$

(b) Polyomino

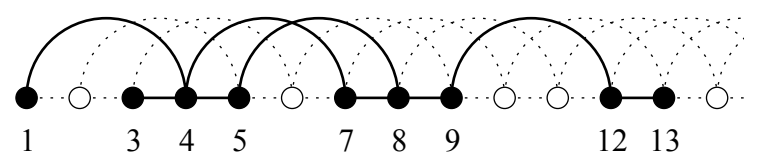

(c) Subgraph of the host graph

Figure 2: A polyomino on the twisted cylinder of width 3.

One can find in [1] formulae for the number of polyominoes on twisted cylinders obtained by building finite automata that model the "growth" of polyominoes on these cylinders, computing the transfer matrices of these automata, and deducing from these matrices the generating functions for the sequences that enumerate polyominoes on the twisted cylinders. This method proves that the formula for the number of polyominoes on a twisted cylinder of any width obeys a linear recurrence. In practice, the size of the involved matrix grows exponentially with $w$ (as do Motzkin numbers), and so, the amount of computations, as well as the orders of the computed formulae, becomes prohibitively large for large values of $w$. For example, for $w=10$, the recurrence includes 2,168 terms, with the largest coefficient being about $6.39 \cdot 10^{129}$ (432 bits).

\section{THE VIDEO}

The video shows the relation between polyominoes and twisted cylinders. First, it defines what polyominoes are, specifies possible uses of them, and mentions the best known lower and upper bounds on the asymptotic growth rate of polyominoes in the plane. It then illustrates the process of counting polyominoes on twisted cylinders by running the algorithm on a twisted cylinder of width 3 . It also demonstrates how this process can be modeled by a finite automaton. Finally, the video shows how we compute the growth rate of polyominoes on twisted cylinders, and concludes with how this implies lower bounds on the respective rate in the plane. Figure 3 shows two snapshots from the video.

The video was produced on a $2.53 \mathrm{GHz}$ DELL 64 processor PC with $4 \mathrm{~GB}$ of RAM. The scenes and animations were designed using the Autodesk Maya 2013 (student version) modeling software. The video was constructed using Windows Live Movie Maker.

\section{REFERENCES}

[1] G. Aleksandrowicz, A. Asinowski, G. Barequet, AND R. BAREQUet, Formulae for polyominoes on twisted cylinders, manuscript.
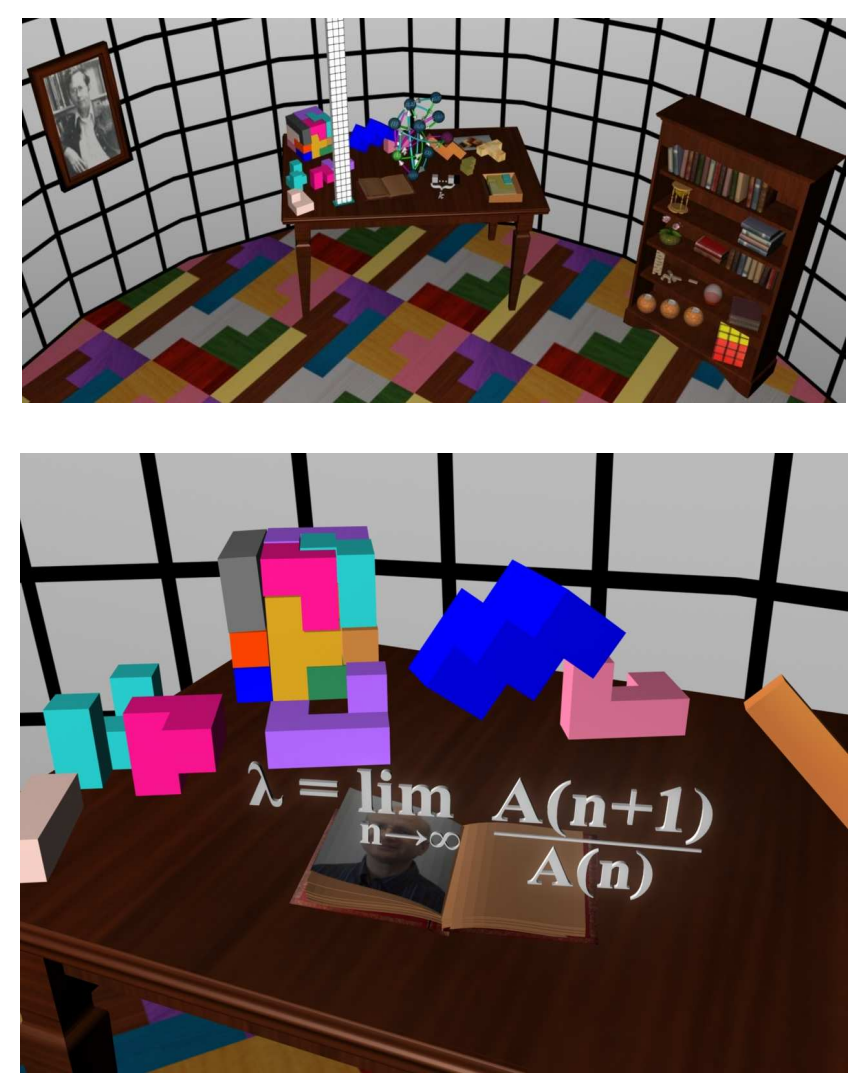

Figure 3: Snapshots from the video.

[2] G. Barequet, M. Moffie, A. Ribó, and G. Rote, Counting polyominoes on twisted cylinders, Integers, 6 (2006), \#A22, 37 pp.

[3] S.R. Broadbent And J.M. Hammersley, Percolation processes: I. Crystals and mazes, Proc. Cambridge Philosophical Society, 53 (1957), 629-641.

[4] D.S. Gaunt, M.F. Sykes, And H. Ruskin, Percolation processes in $d$-dimensions, J. of Physics A: Mathematical and General, 9 (1976), 1899-1911.

[5] I. Jensen, Counting polyominoes: A parallel implementation for cluster computing, Proc. Int. Conf. on Comp. Science, part III, Melbourne, Australia and St. Petersburg, Russia, Lecture Notes in Computer Science, 2659, Springer, 203-212, June 2003.

[6] D.A. KLarner, Cell growth problems, Canadian J. of Mathematics, 19 (1967), 851-863.

[7] D.A. Klarner and R.L. Rivest, A procedure for improving the upper bound for the number of $n$-ominoes, Canadian J. of Mathematics, 25 (1973), $585-602$.

[8] D.H. Redelmeier, Counting polyominoes: Yet another attack, Discrete Mathematics, 36 (1981), 191-203.

[9] N. MAdras, A pattern theorem for lattice clusters, Annals of Combinatorics, 3 (1999), 357-384.

[10] P.J. Peard and D.S. Gaunt, 1/d-expansions for the free energy of lattice animal models of a self-interacting branched polymer, J. of Physics A: Mathematical and General, 28 (1995), 6109-6124. 